\title{
Elevated urinary level of vitamin D-binding protein as a novel biomarker for diabetic nephropathy
}

\author{
XIAO-QIN TIAN ${ }^{1}$, LI-MIN ZHAO ${ }^{2}$, JIA-PU GE ${ }^{2}$, YAN ZHANG $^{3}$ and YAN-CHENG XU ${ }^{1}$ \\ ${ }^{1}$ Department of Endocrinology, Zhongnan Hospital, Wuhan University, Wuhan, Hubei 430071; ${ }^{2}$ Department of Endocrinology, \\ People's Hospital of Xinjiang Uygur Autonomous Region, Urumqi, Xinjiang 830011; ${ }^{3}$ Department of Endocrinology, \\ Traditional Chinese Medicine Hospital, Xinjiang Medical University, Urumqi, Xinjiang 830000, P.R. China
}

Received July 21, 2013; Accepted November 15, 2013

DOI: $10.3892 /$ etm.2013.1426

\begin{abstract}
Improving the early prediction and detection of diabetic nephropathy (DN) remains a great challenge in disease management. The aim of this study was to evaluate the early detection power of urinary vitamin D-binding protein (VDBP) for the diagnosis of DN. Urine samples were obtained from 45 healthy volunteers and 105 diabetic patients with normoalbuminuria (DM group), microalbuminuria (DN1 group) and macroalbuminuria (DN2 group) ( $\mathrm{n}=35$ per group). The VDBP expression patterns in urine from patients and controls were quantified by western blot analysis. The excretion levels of urinary VDBP were quantified with enzyme-linked immunosorbent assay. The quantification results were obtained by correcting for creatinine expression and showed that urinary VDBP levels were significantly elevated in the patients of the DN1 and DN2 groups compared with those of the DM group and normal controls $(1,011.33 \pm 325.30$ and $1,406.34 \pm 239.66$ compared with $466.54 \pm 213.63$ and $125.48 \pm 98.27 \mathrm{ng} / \mathrm{mg}$, respectively) $(\mathrm{P}<0.001)$. Receiver operating characteristic analysis of urinary VDBP levels for the diagnosis of DN rendered an optimum cut-off value of $552.243 \mathrm{ng} / \mathrm{mg}$ corresponding to $92.86 \%$ sensitivity and $85.00 \%$ specificity, which also showed an area under the ROC curve of 0.966 . In conclusion, the findings of the present study suggest that urinary VDBP may be a potential biomarker for the early detection and prevention of DN. Further studies are required to examine the pathogenic mechanisms of elevated VDBP levels and their role in the diagnosis of $\mathrm{DN}$.
\end{abstract}

\section{Introduction}

Diabetes is the fifth leading cause of mortality in the United States and the world prevalence of diabetes is increasing

Correspondence to: Professor Yan-Cheng Xu, Department of Endocrinology, Zhongnan Hospital, Wuhan University, East Lake Road, No. 169, Wuhan, Hubei 430071, P.R. China

E-mail: xyc2007@yahoo.com

Key words: vitamin D binding protein, urine, diabetic nephropathy at an alarming rate with $>1$ million new patients per year diagnosed in the United States $(1,2)$. Furthermore, diabetes results in substantial mortality and morbidity (3). Although the current methods of diagnosis and treatment of diabetes have improved, the long-term prognosis remains poor (4). In addition, there are few effective preventive measures against its development. Epidemiological data suggested that disease prevalence is likely to continue to increase globally without effective prevention and control (5).

Diabetic nephropathy (DN) is one of the most common complications, which results in chronic kidney disease in diabetic patients. It is also one of the causes of increased cardiovascular mortality (4). However, a considerable amount of significant diabetic renal structural injury may occur in absolute clinical silence, which renders diagnosis difficult. Currently, routine kidney biopsy for DN is not permitted in clinical practice, as the procedure is invasive. Microalbuminuria is considered as the best available, non-invasive marker for DN risk, but certain studies have shown it to have inadequate specificity and sensitivity $(6,7)$. Thus, additional studies into novel invasive risk markers are required, and feasible measures for the diagnosis of DN prior to advanced renal dysfunction are considered to be of clinical importance with public health implications.

Vitamin D-binding protein (VDBP), also known as gc-globulin, bonds and transports vitamin $\mathrm{D}$ throughout the body. It also is important in the actin-scavenger system, ensuing immune responses and inflammation processes (8). Clinically, it has been demonstrated that exaggerated excretion of urinary VDBP is associated with tubular dysfunction (9). Therefore, it was hypothesized that the loss of urinary VDBP is likely to be elevated in diabetic patients and particularly accentuated in those patients with DN. In the present study, the early detection and predictive value of urinary VDBP for DN was assessed.

\section{Materials and methods}

Patient selection and urine specimen collection. In this study, 105 Chinese Han individuals with diabetes and 45 healthy volunteers (control group) were recruited from the Traditional Chinese Medicine Hospital attached to Xinjiang Medical University (Urumqi, China) from June 2012 to January 
Table I. Clinical and demographic data for the patients subjected to ELISA analysis.

\begin{tabular}{|c|c|c|c|c|}
\hline Characteristics & Control & DM group & DN1 group & DN2 group \\
\hline Cases (n) & 45 & 35 & 35 & 35 \\
\hline Age (years) & $60.89 \pm 13.92$ & $64.63 \pm 11.76$ & $64.49 \pm 11.78$ & $64.80 \pm 12.98$ \\
\hline \multicolumn{5}{|l|}{ Gender (n) } \\
\hline Male & 31 & 22 & 18 & 21 \\
\hline Female & 14 & 13 & 17 & 4 \\
\hline \multicolumn{5}{|l|}{ Smoking (n) } \\
\hline Yes & 20 & 14 & 17 & 19 \\
\hline No & 25 & 21 & 18 & 16 \\
\hline \multicolumn{5}{|l|}{ Diabetes (n) } \\
\hline Type 1 & 0 & 5 & 5 & 7 \\
\hline Type 2 & 0 & 30 & 30 & 28 \\
\hline Diabetic duration (years) & 0 & $11.43 \pm 7.83$ & $12.77 \pm 8.65$ & $11.57 \pm 6.45$ \\
\hline Systolic BP (mmHg) & $115 \pm 8.32$ & $120 \pm 9.59$ & $134 \pm 8.76^{*}$ & $143 \pm 10.32^{*}$ \\
\hline Serum creatinine $(\mu \mathrm{mol} / \mathrm{l})$ & $99.84 \pm 24.23$ & $92.43 \pm 36.65$ & $107.34 \pm 46.62$ & $142.46 \pm 55.10^{*, *, * * * *}$ \\
\hline UACR (mg/g) & $10.52 \pm 2.78$ & $13.08 \pm 4.11$ & $134.66 \pm 47.2^{*, * *}$ & $1603.09 \pm 544.60^{*, * *, * * *}$ \\
\hline Taking ACEI (n) & 0 & 12 & 15 & 14 \\
\hline Taking ARB (n) & 0 & 13 & 10 & 12 \\
\hline Insulin therapy (n) & 0 & 35 & 35 & 35 \\
\hline
\end{tabular}

Measurement data are presented as the mean \pm standard deviation. ${ }^{*} \mathrm{P}<0.05$ compared with the control group; ${ }^{* *} \mathrm{P}<0.05$ compared with the DM group; and ${ }^{* * *} \mathrm{P}<0.05$ compared with the DN1 group. DM, UACR $<30 \mathrm{mg} / \mathrm{g}$; DN, diabetes nephropathy; DN1, with microalbuminuria $30<\mathrm{UACR}<300 \mathrm{mg} / \mathrm{g}$; DN2, with microalbuminuria UACR $>300 \mathrm{mg} / \mathrm{g}$; UACR: urinary albumin:creatinine ratio; BP, blood pressure; ACEI, angiotensin-converting enzyme inhibitor; ARB, angiotensin-receptor blocker; ELISA, enzyme-linked immunosorbent assay.

2013. The patients were divided into three groups according to the value of the urinary albumin:creatinine $(\mathrm{Cr})$ ratio (UACR): DM group without nephropathy and albuminuria (UACR<30 mg/g, $\mathrm{n}=35$ ), early DN group (DN1) with microalbuminuria $(30 \leq \mathrm{UACR}<300 \mathrm{mg} / \mathrm{g}, \mathrm{n}=35)$ and overt $\mathrm{DN}$ group (DN2) with macroalbuminuria (UACR $\geq 300 \mathrm{mg} / \mathrm{g}, \mathrm{n}=35$ ). All study subjects were $>18$ years old and blood pressure was maintained at $140 / 90 \mathrm{mmHg}$ by the use of angiotensin-receptor blockers or angiotensin-converting enzyme inhibitors. Moreover, blood glucose was controlled with human insulin. Within the 2 weeks prior to urine collection, patients did not receive additional medicine and the controls did not receive any medication. Patients were excluded if they showed the following: Liver diseases, autoimmune diseases, inflammatory diseases, pregnancy, urinary system disorders, tumors, infections, decompensated heart failure, cardiovascular events within 6 months, hematological diseases and known renal diseases other than DN. Studies were approved by the local ethics committee of the Traditional Chinese Medicine Hospital attached to Xinjiang Medical University (Urumqi, China). All patients were informed about the purpose of the study and gave their written consent.

Urine specimen collection. The second voided clean-catch urine samples from all patients were collected early in the morning. Each urine sample $(20 \mathrm{ml})$ was directly collected into a sterile plastic tube and then immediately centrifuged at
$2,500 \mathrm{x}$ g for $10 \mathrm{~min}$ at $4^{\circ} \mathrm{C}$ to remove cell debris and particulate matter. The supernatant was stored at $-80^{\circ} \mathrm{C}$ for further analysis. Repeated freeze-thaw cycles were avoided. Clinical data of all patients were also collected.

Western blot analysis. The stored supernatant was used for western blot analysis after measuring the protein concentrations. The urine samples were first thawed on ice, adding $1 \mathrm{mmol} / 1$ phenylmethanesulfonyl fluoride (Sigma, St. Louis, MO, USA), and then centrifuged using Centricon Plus-20, 10,000 MWCO devices (Millipore, Bedford, MA, USA). Then the concentrated urinary proteins were precipitated using a ReadyPrep 2-D clean up kit (GE Healthcare, Piscataway, NJ, USA) to remove other interfering components according to the manufacturer's instructions The concentrations of urinary proteins were determined by using the Bradford protein assay kits (GE Healthcare). The samples examined were from patients from the DM, DN1, DN2 and control groups $(n=8$ per group). A total of $20 \mu \mathrm{g}$ prepared proteins isolated from the urine samples were electrophoresed on a $12 \%$ sodium dodecyl sulfate-polyacrylamide gel. The proteins were then transferred onto polyvinylidene fluoride membranes (Immobilon P; Millipore, Billerica, MA, USA). The membranes were blocked for $1 \mathrm{~h}$ at $37^{\circ} \mathrm{C}$ in a solution of TBS containing $5 \%$ non-fat milk powder and $0.1 \%$ Tween-20 (TBS-T) and then incubated overnight at $4{ }^{\circ} \mathrm{C}$ with rabbit monoclonal primary antibody against human VDBP (diluted 1:1,000; Abcam, Cambridge, 
UK). Membranes were washed three times for $10 \mathrm{~min}$ in TBS-T and then incubated with horseradish peroxidase horseradish-coupled goat anti-rabbit IgG (Beijing Zhongshan Biotechnology Co. Ltd., Beijing, China) at a 1:500 dilution at room temperature for $1 \mathrm{~h}$. The proteins were detected using an enhanced chemiluminescence detection system (ECL-Direct systems RPN3000; Pierce Biotechnology, Inc., Rockford, IL, USA). Triplicate gel images of identical samples were used for analysis. It was quantified by strip densitometry.

Enzyme-linked immunosorbent assay(ELISA). All the samples were centrifuged at $3,500 \mathrm{x}$ g for $5 \mathrm{~min}$ at $4^{\circ} \mathrm{C}$ to remove interfering matter prior to ELISA analysis. The concentrations of VDBP in the urine samples were measured with a Human Vitamin D BP Quantikine ELISA kit (DVDBP0; R\&D Systems, Minneapolis, MN, USA). The assay was performed according to the instructions recommended by the manufacturer. The standard curve was created using the lyophilized human VDBP standard preparation supplied with the assay. Following the colorimetric reaction, the optical density (OD) readings were converted to concentrations in $\mathrm{ng} / \mathrm{ml}$ based on quantification of the OD at $450 \mathrm{~nm}$ using an eight-channel spectrophotometer (Fast model; BD Biosciences, Franklin Lakes, NJ, USA). Measured VDBP levels ranged from 0 to $250 \mathrm{ng} / \mathrm{ml}$. Urine Cr levels were measured at the Department of Clinical Laboratory, Traditional Chinese Medicine Hospital attached to Xinjiang Medical University (Urumqi, China). The levels of VDBP were normalized according to urine $\mathrm{Cr}$ concentrations so as to avoid the influence of urine volume and presented as VDBP:Cr ratio (VDBP-Cr; ng/mg of Cr) (10). Every sample was tested in duplicate.

Statistical analysis. All data were collected and presented as the mean \pm standard deviation. The differences among groups were compared with Student's t-test between two groups or one-way analysis of variance for three groups. Receiving operating curve (ROC) analyses were used to explore the diagnostic performance of urinary VDBP:Cr over a range of possible clinical results on the basis of estimating the sensitivity versus its false-positive rate at optimal cut-offs $(10,11)$. The best statistical cut-off value of VDBP:Cr was defined, which means the point at which the sum of sensitivity and specificity is more than that at other points. Pearson's correlation coefficient analysis was employed to explore the association between the VDBP:Cr and the clinical features of patients. Multivariate logistical regression analysis was utilized to determine the risk factors for DN. All statistical analyses were performed with SPSS software, version 13.0 (SPSS, Chicago, IL, USA). All tests were two tailed and $\mathrm{P}<0.05$ was considered to indicate a statistically significant difference.

\section{Results}

Clinical characteristics of patients. The clinical characteristics of all patients are shown in Table I. Samples from 35 DM, $35 \mathrm{DN} 1$ and $35 \mathrm{DN} 2$ patients and 45 controls were collected for ELISA analysis. Analysis of the patient data indicated that there were no statistically significant differences in the majority of clinical characteristics (e.g. age, gender, smoking and duration of diabetes) among the four groups. However,
A

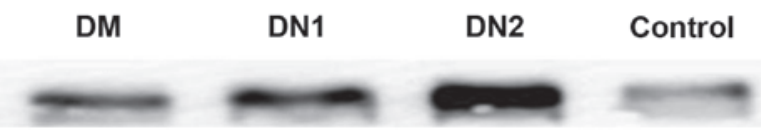

B

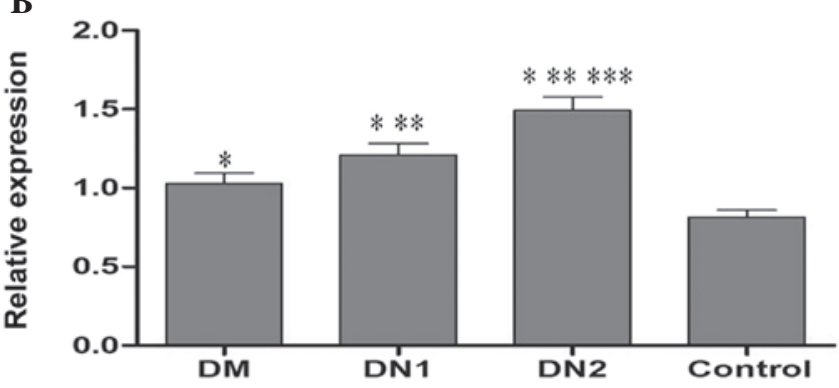

Figure 1. VDBP expression levels were verified in the urine samples (A) Western blot analysis of VDBP expression in urine samples of DM, DN1, DN2 and control groups. (B) VDBP protein expression levels in individual urine samples was calculated according to the immunosignals quantified by densitometric scanning. Data are expressed as the mean \pm standard deviation from three independent experiments. ${ }^{*} \mathrm{P}<0.05$ compared with that of the control group; ${ }^{* *} \mathrm{P}<0.05$ compared with that of the DM group and ${ }^{* * *} \mathrm{P}<0.05$ compared with that of the DN1 group. DM, UACR $<30 \mathrm{mg} / \mathrm{g}$; DN, diabetic nephropathy; DN1, with microalbuminuria $30<\mathrm{UACR}<300 \mathrm{mg} / \mathrm{g}$; DN2, with microalbuminuria UACR>300 mg/g; UACR, urinary albumin:creatinine ratio; VDBP, vitamin D binding protein; UACR, urine:creatinine ratio.

significant differences were identified in serum $\mathrm{Cr}$, UACR and systolic blood pressure between certain groups (serum-Cr: $\mathrm{DN} 2$ vs. control $\mathrm{P}=0.025, \mathrm{DN} 2$ vs. $\mathrm{DM}: \mathrm{P}=0.012 \mathrm{DN} 2$ vs. DN1: $\mathrm{P}=0.034$, UACR-DN1 vs. control: $\mathrm{P}<0.001$, DN1 vs. DM: $\mathrm{P}<0.001$; DN2 vs. control $\mathrm{P}<0.001$, DN2 vs. $\mathrm{DM}$ : $\mathrm{P}<0.001$, DN2 vs. DN1: $\mathrm{P}<0.001$; systolic blood pressure-DN1 vs. control: $\mathrm{P}=0.021$, $\mathrm{DN} 2$ vs. control: $\mathrm{P}=0.014)$. Additionally, as the patients in each experimental group had taken similar medications, the effect of taking medicine on the results was ignored.

Analysis of urinary VDBP by western blotting. To verify the expression of urinary VDBP in individual urine samples by western blotting, 32 samples from the DM, DN1, DN2 and control groups ( $n=8$ per group) were randomly selected. The results demonstrated that urinary VDBP levels were significantly upregulated in the DM group compared with that of the control group (Fig. 1). Furthermore, the levels of urinary VDBP were significantly elevated in the DN groups compared with those of the DM and control groups (Fig. 1).

Detection of urinary VDBP expression. To explore the changes of urinary VDBP expression in DN patients, ELISA analysis was conducted on the 150 urine samples from the DM, DN1, DN2 and control groups.

The levels of urinary VDBP were significantly higher in patients than in controls $(961.41 \pm 542.77$ versus $125.48 \pm 98.27 \mathrm{ng} / \mathrm{mg}, \mathrm{P}<0.001$; Fig. 2A). After division of the patients into groups by UACR, it was indicated that the expression levels of urinary VDBP were significantly higher in the DN1 and DN2 groups than in the DM group $(1,011.33 \pm 325.30$ and $1,406.34 \pm 239.66$ versus $466.54 \pm 213.63 \mathrm{ng} / \mathrm{mg}$, respec- 


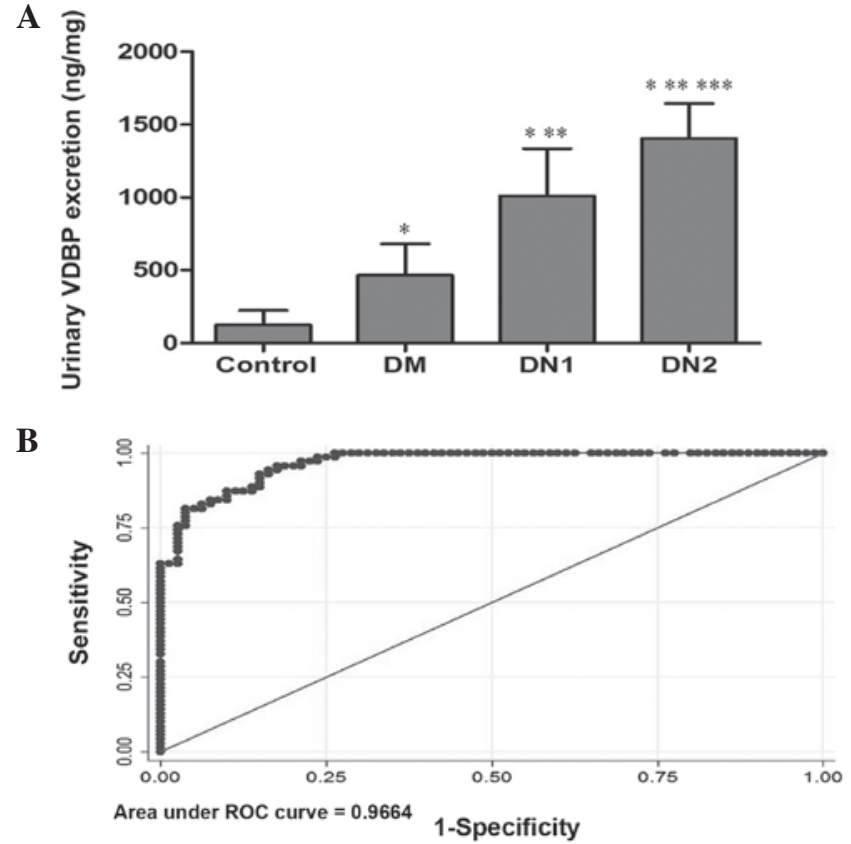

Figure 2. Evaluation of urinary VDBP levels as a biomarker for DN. (A) ELISA quantification of VDBP levels in the urine of DM, DN1, DN2 and control groups. Data are expressed as the mean \pm standard deviation. ${ }^{*} \mathrm{P}<0.05$ compared with the control group; ${ }^{* *} \mathrm{P}<0.05$ compared with the DM group; and ${ }^{* * *} \mathrm{P}<0.001$ compared with the DN1 group. (B) ROC curve of urinary VDBP as a biomarker of early detection and prevention for DN was based on an optimum cut-off value of $552.243 \mathrm{ng} / \mathrm{mg}$ corresponding to $92.86 \%$ sensitivity and $85.00 \%$ specificity. The area under the ROC curve was 0.966 (95\% CI, 0.924-0.989). VDBP, vitamin D binding protein; DN, diabetes nephropathy; $\mathrm{DM}, \mathrm{UACR}<30 \mathrm{mg} / \mathrm{g}$; DN, diabetic nephropathy; DN1, with microalbuminuria $30<\mathrm{UACR}<300 \mathrm{mg} / \mathrm{g}$; DN2, with microalbuminuria UACR $>300 \mathrm{mg} / \mathrm{g}$; UACR, urinary albumin:creatinine; ROC, receiver operating characteristics.

tively; $\mathrm{P}<0.001)$. A significant difference was also observed between the DN2 and DN1 groups $(\mathrm{P}<0.001$; Fig. $2 \mathrm{~A}$ and Table II).

Correlation and multivariate logistical regression analyses. Additional subgroup analyses were performed to clarify the correlation between urinary VDBP levels and clinical characteristics of the patients (Table II). No significant association was observed between the urinary loss of VDBP and the clinical features of DM patients, such as age, gender, smoking, duration of DM and hypertension, with the exception of DN and renal dysfunction. The enhanced excretion levels of urinary VDBP were significantly higher in patients with renal dysfunction than in the patients without dysfunction $(1,263.15 \pm 531.33$ versus $790.27 \pm 473.08 \mathrm{ng} / \mathrm{mg}$; P<0.001). Furthermore, higher urinary VDBP concentrations were detected in the DN1 and DN2 groups than in the DM group $(\mathrm{P}<0.001)$. The Spearman rank correlation was 0.707 , which indicated that the levels of urinary VDBP had a strong positive correlation with the development of DN. Also, multivariate logistical regression analysis was conducted to assess the independent risk factors for DN. The results suggested that serum $\mathrm{Cr}$ may be an independent predictor of $\mathrm{DN}(\mathrm{OR}=1.29,95 \%$ confidence interval, 1.02-1.57; $\mathrm{P}=0.04)$.

Evaluation of urinary VDBP as a biomarker for DN. Following quantitative analysis of 150 urine samples by
Table II. Correlation between levels of urinary VDBP and clinical features of diabetic patients.

\begin{tabular}{|c|c|c|c|}
\hline Clinical features & No. & $\begin{array}{l}\text { Urinary VDBP } \\
\text { levels (ng/mg) }\end{array}$ & P-value \\
\hline \multicolumn{4}{|l|}{ Age } \\
\hline$>65$ years & 52 & $1009.52 \pm 533.27$ & \multirow[t]{2}{*}{0.37} \\
\hline$\leq 65$ years & 53 & $914.20 \pm 552.91$ & \\
\hline \multicolumn{4}{|l|}{ Gender } \\
\hline Female & 44 & $1001.44 \pm 491.33$ & \multirow[t]{2}{*}{0.52} \\
\hline Male & 61 & $932.53 \pm 579.32$ & \\
\hline \multicolumn{4}{|l|}{ Hypertension } \\
\hline With & 36 & $1050.86 \pm 426.16$ & \multirow[t]{2}{*}{0.22} \\
\hline Without & 69 & $914.74 \pm 592.15$ & \\
\hline \multicolumn{4}{|l|}{ Smoking } \\
\hline Yes & 50 & $1043.62 \pm 618.68$ & \multirow[t]{2}{*}{0.14} \\
\hline No & 55 & $886.66 \pm 456.19$ & \\
\hline \multicolumn{4}{|l|}{ Renal dysfunction } \\
\hline With & 38 & $1263.15 \pm 531.33$ & \multirow[t]{2}{*}{$<0.001$} \\
\hline Without & 67 & $790.27 \pm 473.08$ & \\
\hline \multicolumn{4}{|l|}{ Diabetes } \\
\hline Type 1 & 18 & $1083.46 \pm 582.21$ & \multirow[t]{2}{*}{0.30} \\
\hline Type 2 & 87 & $936.15 \pm 534.31$ & \\
\hline \multicolumn{4}{|l|}{ Diabetic duration } \\
\hline$>12$ years & 43 & $975.74 \pm 543.32$ & \multirow[t]{2}{*}{0.82} \\
\hline$\leq 12$ years & 62 & $951.46 \pm 546.60$ & \\
\hline DM group & 35 & $466.54 \pm 213.63$ & $<0.001$ \\
\hline DN1 group & 35 & $1011.33 \pm 325.30$ & \\
\hline DN2 group & 35 & $1406.34 \pm 539.66$ & \\
\hline
\end{tabular}

$\mathrm{DM}, \mathrm{UACR}<30 \mathrm{mg} / \mathrm{g}$; DN, diabetic nephropathy; DN1, early DN with microalbuminuria $(30<\mathrm{UACR}<300 \mathrm{mg} / \mathrm{g}) ; \mathrm{DN} 2$, overt $\mathrm{DN}$ group with macroalbuminuria (UACR $>300 \mathrm{mg} / \mathrm{g}$ ). UACR, urinary albumin:creatinine ratio; VDBP, vitamin D binding protein.

ELISA, ROC curves were used to assess the potential utility of urinary VDBP detection in patients with DN. The area under the ROC curve of urine VDBP levels for the diagnosis of DN was 0.966 (95\% CI, 0.924-0.989). The analysis rendered an optimum cut-off value of $552.243 \mathrm{ng} / \mathrm{mg}$ corresponding to $92.86 \%$ sensitivity and $85.00 \%$ specificity (Fig. 2B).

\section{Discussion}

Improving the early prediction and detection of $\mathrm{DN}$ remains a great challenge in disease management $(13,14)$. Improving the predictive ability of testing would greatly benefit the treatment of patients with DN and facilitate the monitoring of the condition. To explore whether urine VDBP levels may be a novel non-invasive biomarker for DN, the results of the present study demonstrated that the expression level of urinary VDBP was highly upregulated in patients with DN. Furthermore, the levels of VDBP were measured in the urine samples from the control, DM and DN groups. 
VDBP is a 58-kDa glycoprotein and is present in the serum at a concentration of $300-600 \mathrm{mg} / \mathrm{ml}(15)$. It serves as the main carrier protein for vitamin D in the bloodstream. VDBP is important in the bioavailability of active 1,25-dihydroxyvitamin $\mathrm{D}\left(1,25(\mathrm{OH})_{2} \mathrm{D}\right)$ and its precursor 25 -hydroxyvitamin $\mathrm{D}$ (25OHD) $(16,17)$. The transportation of vitamin D by VDBP is important for the function of a wide variety of tissues and changes in VDBP activity result in the development of a number of diseases (8).

In addition to its transport function, VDBP is the parent molecule of VDBP-maf (macrophage activating factor). VDBP-maf is the product of the selectively deglycosylated form of VDBP and has been demonstrated to be a potent antiangiogenic and antitumorigenic molecule (18). Such functions would greatly benefit the regulation of the growth of cancer cells and protection against certain immune disorders (19). These important and diverse properties of VDBP have been suggested in previous studies with regard to a number of tumor types $(20,21)$. Moreover, VDBP is important in the actin scavenger system and inflammation processes (8). Studies concerning the actions of VDBP in the kidney have received increased attention and have suggested that VDBP is vital in the endocrine biosynthetic process of $1,25(\mathrm{OH})_{2} \mathrm{D}$ within renal proximal tubules; in this process, $25 \mathrm{OHD}$ binds to VDBP and the complex is actively recovered from glomerular filtrate through megalin-mediated receptor endocytosis $(22,23)$.

In the present study, following quantitative measurements of 150 urine samples with ELISA, it was identified that the VDBP expression levels were significantly higher in the urine samples from patients with DN than in the urine from the DM and control groups. Furthermore, a strong positive correlation was observed between the urinary VDBP levels and the development of DN. ROC analysis rendered that an optimum cut-off value of urinary VDBP of $552.243 \mathrm{ng} / \mathrm{mg}$ corresponding to $92.86 \%$ sensitivity and $85.00 \%$ specificity is appropriate for detecting DN. On the basis of these analyses, urinary VDBP was indicated to be a potential biomarker for the early detection and prevention of DN.

The reasons underlying the enhanced excretion of urinary VDBP in patients with DN remain unclear. One possible explanation is that elevated urinary VDBP levels may be associated with renal tubular damage in DN patients $(24,25)$. Renal tubular epithelial cell damage becomes increasingly severe as DN develops. In a previous study, increased excretion of urinary VDBP was observed following long-term cadmium exposure, and it was suggested that the marked loss of VDBP in the urine may be linked to renal tubular dysfunction and bone lesions in the inhabitants of cadmiumpolluted areas (26). In addition, it has been demonstrated that the presence of vitamin D deficiency or insufficiency in patients with diabetes is independently associated with the development of DN. Moreover, exaggerated urinary excretion of VDBP was observed in patients with Type I diabetes, which contributed mechanistically to vitamin D deficiency in this disease $(9,27,28)$. Therefore, a further possibility for the elevated urinary VDBP levels identified in the present study may be associated with the relatively lower serum vitamin D levels. Further studies are required to clarify the role of VDBP in the pathogenesis of $\mathrm{DN}$.
Clinically, there have been studies on aspects of other diseases, which demonstrated the increased urinary excretion of VDBP. Recently, Mirković et al indicated that the urinary excretion of VDBP may be a novel urinary biomarker of tubulointerstitial damage (29). Cho et al demonstrated that the urinary VDBP loss was significantly elevated in patients with endometriosis than those without. However, the authors suggested that urinary VDBP has limited value as a potential early diagnostic biomarker for endometriosis (10). A study by Zoidakis et al identified that the reduction of VDBP levels in the urine of patients with invasive bladder cancer was significant (30), which is consistent with the findings by Li et al (8). Moreover, Li et al also demonstrated that the expression levels of urinary VDBP were positively associated with the pathological classification of bladder cancer (9). Their results suggested that urinary VDBP may be a potential non-invasive biomarker for the early diagnosis and effective surveillance of bladder cancer (8). In the present study, to the best of our knowledge, it was demonstrated for the first time that increased urinary VDBP levels occurred in patients with DN, and there was a strong positive association between urinary VDBP levels and the development of DN.

An important limitation of the present study regarding the specificity of this biomarker should be considered when urinary VDBP detection is used for early prevention of DN. It has been demonstrated that urinary VDBP levels are closely associated with renal dysfunction. In the present study, urine samples were collected from patients with DN only, but not from patients with additional nephropathies. This may have caused an overestimation of the specificity of VDBP as a biomarker for the detection of DN. Therefore, further studies including a larger sample and analyses from patients with various types of non-diabetic nephropathy are required to clarify this issue.

In conclusion, the current study demonstrated that urinary VDBP levels were significantly elevated in patients with DN. Moreover, a strong positive correlation was observed between the expression level of urinary VDBP and the development of DN. Thus, the findings indicate that urinary VDBP levels are a potential biomarker for the early detection and prevention of DN. Further studies are warranted to examine the pathogenic mechanisms of elevated VDBP and its role in the diagnosis of DN.

\section{References}

1. Hu FB, Manson JE, Stampfer MJ, Colditz G, Liu S, Solomon CG and Willett WC: Diet, lifestyle, and the risk of type 2 diabetes mellitus in women. N Engl J Med 345: 790-797, 2001.

2. Pittas AG, Lau J, Hu FB and Dawson-Hughes B: The role of vitamin $\mathrm{D}$ and calcium in type 2 diabetes. A systematic review and meta-analysis. J Clin Endocrinol Metab 92: 2017-2029, 2007.

3. Iso H: Changes in coronary heart disease risk among Japanese. Circulation 118: 2725-2729, 2008.

4. Valmadrid CT, Klein R, Moss SE and Klein BE: The risk of cardiovascular disease mortality associated with microalbuminuria and gross proteinuria in persons with older-onset diabetes mellitus. Arch Intern Med 160: 1093-1100, 2000.

5. Wild S, Roglic G, Green A, Sicree R and King H: Global prevalence of diabetes: estimates for the year 2000 and projections for 2030. Diabetes Care 27: 1047-1053, 2004.

6. Tabaei BP, Al-Kassab AS, Ilag LL,Zawacki CM and Herman WH: Does microalbuminuria predict diabetic nephropathy? Diabetes Care 24: 1560-1566, 2001. 
7. Jiang H, Guan G, Zhang R, Liu G, Cheng J, Hou X and Cui Y: Identification of urinary soluble E-cadherin as a novel biomarker for diabetic nephropathy. Diabetes Metab Res Rev 25: 232-241, 2009.

8. Li F, Chen DN, He CW, Zhou Y, Olkkonen VM, He N, Chen W, Wan P, Chen SS, Zhu YT, Lan KJ and Tan WL: Identification of urinary Gc-globulin as a novel biomarker for bladder cancer by two-dimensional fluorescent differential gel electrophoresis (2D-DIGE). J Proteomics 77: 225-236, 2012.

9. Thrailkill KM, Jo CH, Cockrell GE, Moreau CS and Fowlkes JL: Enhanced excretion of vitamin D binding protein in type 1 diabetes: a role in vitamin D deficiency? J Clin Endocrinol Metab 96: 142-149, 2011.

10. Cho S, Choi YS, Yim SY, Yang HI, Jeon YE, Lee KE, Kim H, Seo SK and Lee BS: Urinary vitamin D-binding protein is elevated in patients with endometriosis. Hum Reprod 27: 515-522, 2012.

11. Hanley JA and McNeil BJ: The meaning and use of the area under a receiver operating characteristic (ROC) curve. Radiology 143: 29-36, 1982.

12. Jiang H, Guan G, Zhang R, Liu G, Liu H, Hou X and Cheng J: Increased urinary excretion of orosomucoid is a risk predictor of diabetic nephropathy. Nephrology (Carlton) 14: 332-337, 2009.

13. Dihazi H and Müller GA: Urinary proteomics: a tool to discover biomarkers of kidney diseases. Expert Rev Proteomics 4: 39-50, 2007.

14. Varghese SA, Powell TB, Budisavljevic MN, Oates JC, Raymond JR, Almeida JS and Arthur JM: Urine biomarkers predict the cause of glomerular disease. J Am Soc Nephrol 18: 913-922, 2007.

15. Cooke NE and David EV: Serum vitamin D-binding protein is a third member of the albumin and alpha fetoprotein gene family. J Clin Invest 76: 2420-2424, 1985.

16. Chun RF, Peercy BE, Adams JS and Hewison M: Vitamin D binding protein and monocyte response to 25-hydroxyvitamin $\mathrm{D}$ and 1,25-dihydroxyvitamin D: analysis by mathematical modeling. PLoS One 7: e30773, 2012.

17. Christakos S, Ajibade DV, Dhawan P, Fechner AJ and Mady LJ: Vitamin D: metabolism. Endocrinol Metab Clin North Am 39: 243-253, 2010.

18. Yamamoto $\mathrm{N}$ and Naraparaju VR: Vitamin D3-binding protein as a precursor for macrophage activating factor in the inflammation-primed macrophage activation cascade in rats. Cell Immunol 170: 161-167, 1996.

19. Gregory KJ, Zhao B, Bielenberg DR, Dridi S, Wu J, Jiang W, Huang B, Pirie-Shepherd S and Fannon M: Vitamin D binding protein-macrophage activating factor directly inhibits proliferation, migration, and uPAR expression of prostate cancer cells. PLoS One 5: e13428, 2010.
20. Yamamoto N, Naraparaju VR and Asbell SO: Deglycosylation of serum vitamin D3-binding protein leads to immunosuppression in cancer patients. Cancer Res 56: 2827-2831, 1996.

21. Yamamoto N, Suyama H, Nakazato H, Yamamoto N and Koga Y: Immunotherapy of metastatic colorectal cancer with vitamin D-binding protein-derived macrophage-activating factor, GcMAF. Cancer Immunol Immunother 57: 1007-1016, 2008.

22. Nykjaer A, Dragun D, Walther D, Vorum H, Jacobsen C, Herz J, Melsen F, Christensen EI and Willnow TE: An endocytic pathway essential for renal uptake and activation of the steroid 25-(OH) vitamin D3. Cell 96: 507-515, 1999.

23. Zehnder D, Bland R, Williams MC, McNinch RW, Howie AJ, Stewart PM and Hewison M: Extrarenal expression of 25-hydroxyvitamin $\mathrm{D}_{3}$-1 $\alpha$-hydroxylase. J Clin Endocrinol Metab 86: 888-894, 2001

24. Colston K, Williams NJ and Cleeve HJ: Studies on vitamin $\mathrm{D}$ binding protein in the nephrotic syndrome. Clin Chem 31: 718-721, 1985.

25. Vilasi A, Cutillas PR, Maher AD, Zirah SF, Capasso G, Norden AW, Holmes E, Nicholson JK and Unwin RJ: Combined proteomic and metabonomic studies in three genetic forms of the renal Fanconi syndrome. Am J Physiol Renal Physiol 293: F456-F467, 2007.

26. Uchida M, Teranishi H, Aoshima K, Katoh T, Kasuya M and Inadera $\mathrm{H}$ : Elevated urinary levels of vitamin D-binding protein in the inhabitants of a cadmium polluted area, Jinzu River basin, Japan. Tohoku J Exp Med 211: 269-274, 2007.

27. Svoren BM, Volkening LK, Wood JR and Laffel LM: Significant vitamin D deficiency in youth with type 1 diabetes mellitus. J Pediatr 154: 132-134, 2009.

28. Tahrani AA, Ball A, Shepherd L, Rahim A, Jones AF and Bates A: The prevalence of vitamin D abnormalities in South Asians with type 2 diabetes mellitus in the UK. Int J Clin Pract 64: 351-355, 2010.

29. Mirković K, Doorenbos CR, Dam WA, Lambers Heerspink HJ, Slagman MC, Nauta FL, Kramer AB, Gansevoort RT, van den Born J, Navis G and de Borst MH: Urinary vitamin D binding protein: a potential novel marker of renal interstitial inflammation and fibrosis. PLoS One 8: e55887, 2013.

30. Zoidakis J, Makridakis M, Zerefos PG, Bitsika V, Esteban S, Frantzi M, Stravodimos K, Anagnou NP, Roubelakis MG, Sanchez-Carbayo M and Vlahou A: Profilin 1 is a potential biomarker for bladder cancer aggressiveness. Mol Cell Proteomics 11: M111.009449, 2012. 